\title{
Balance and prevention of fall among elderly
}

\begin{abstract}
Background: Currently, the risk of fall is high among elderly population in Saudi Arabia. Nowadays, the interest is directed towards prevention of fall through the prospective research studies in order to minimize the risk of falls.

Objective: To determine the predictors of risk of falls among older subjects in Saudi Arabia. Materials and

Methods: Six hundred elderly subjects, their age ranged from 50-70 years were enrolled in the present study. All participants were asked to answer specific questionnaires, perform Berg Balance Scale (BBS), which is a test for balance and motor skills in addition to conducting the Amer Dizziness Diagnostic Scale (ADDS).

Results: The majority (about 64\%) of the participants have low record $(<45)$ in score in Berg Balance Score which indicates poor balance performance. Regarding the relation between the value the age and the Berg Balance Score, there was strong negative correlation between them $(\mathrm{r}=-0.87, \mathrm{p}<0.001)$. The number of medication intake had strong negative correlation with the Berg Balance Score $(r=-0.85, p<0.001)$. Regarding, the subjects activity level (sedentary vs. active), there was a strong direct correlation between the activity level and the Berg Balance Score $(r=0.76, p<0.001)$. Moreover, there was a strong direct correlation between the subject's ambulatory status and the Berg Balance Score $(r=0.78, p<0.001)$. Results showed, the ADDS revealed only 200 elderly subjects had dizziness out of the 600 elderly subjects participated in this study and the individual number of each type of dizziness were as follows: Benign Paroxysmal Positional vertigo (BPPV) 99 [16.5\%], Unilateral Vestibular Hypo-function (UVH) 44 [7.3\%], Cervico-genic Dizziness (CGD) 26 [4.3\%] and Central Mediated Problem (CM) 31 [5.2\%]. In addition, the results proved that the ADDS strongly correlated with the "true-positive" and "true-negative" results $(\mathrm{r}=0.95, \mathrm{p}<0.05)$. A stepwise linear regression was conducted and the results indicated that ADDS was a significant predictor of "true-positive" and "true-negative" results $(\mathrm{R} 2=0.90, \mathrm{p}<0.05)$
\end{abstract}

Conclusion: Reduction of the controllable risk factors of fall is highly recommended in order to reduce the incidence of fall among elderly. Based on the results, it is also recommended to incorporate the preventive measures of fall in elderly as one of the first priority in all the geriatric care centers across Saudi Arabia. In addition, it is recommended to have more research about the quality of life among elderly in Saudi Arabia.

Keywords: balance, fall, elderly, dizziness, dizziness diagnostic scale, berg balance scale
Volume 3 Issue I - 2018

\author{
Amer Abdalagleel Alsaif,' Samira Ahmed \\ Alsenany ${ }^{2}$ \\ 'Department of Physical Therapy, Faculty of Applied Medical \\ Sciences, King Abdulaziz University, Saudi Arabia \\ ${ }^{2}$ Department of Community Health, Faculty of Nursing, King \\ Abdulaziz University, Saudi Arabia
}

Correspondence: Amer Abdalagleel Alsaif, Department of Physical Therapy, Faculty of Applied Medical Sciences, King Abdulaziz University, Saudi Arabia, Tel 00966554734658222, Email profalsaif@hotmail.com

Received: January 29, 2018 | Published: February 06, 2018
Abbreviations: ADDS, amer dizziness diagnostic scale; BPPV, benign paroxysmal positional vertigo; UVH, unilateral vestibular hypo-function; CGD, cervico-genic dizziness; $\mathrm{CM}$, central mediated problem; VNG, video nystagmography; ENG, electro nystagmography; IPAQ, international physical activity questionnaire; UVH, unilateral vestibular hypo-function; BPPV, benign paroxysmal positional vertigo

\section{Introduction}

Globally, the number of older subjects reached about 600 million, and this number is expected to be about 1.2 billion by 2025 and to reach 2 billion by $2050 .^{1,2}$ Currently, about $70 \%$ of this number live in the third world countries. ${ }^{3}$ The current number of older subjects in Saudi Arabia is one million which equal about $4 \%$ of the whole population and this number is continually rising and their number is expected to be 2.5 million double by 2020 which equals about $7 \%$ of the whole population. ${ }^{4,5}$ Falls in older adults are a major public health problem; affecting 1 in 3 persons aged 65 . These falls may lead to physical injuries and subsequent loss of independence, whereas sometimes which may lead to even death. ${ }^{6}$

Balance deficit is usually multifactorial as it may be related to vestibular, sensory, visual systems dysfunction, musculoskeletal and central processing impairment or combination of more than one of these factors. ${ }^{7}$ Accurate identification of patient impairment is extremely important to provide the proper treatment, restore function and prevent further deterioration of vestibular function. ${ }^{8}$ The initial diagnosis of vestibular dysfunction was based upon the results of routine methods as caloric test, nystagmus test and head thrust test. ${ }^{9}$ Currently, with the evolution of high-tech advances, there are many laboratory tests for evaluation of the vestibular and balance systems alongside of clinical history; these includes, video nystagmography (VNG) recording for eye examinations, caloric test and rotary chair testing and electro nystagmography (ENG). Traditionally, electro nystagmography was considered the "gold standard" for assessment of dizzy patients..$^{10,11}$ 
Although there are multiple tests in existence for documenting vestibular dysfunction, the cost and the time required to perform these tests often prevent their use in general clinics. As the causes of dizziness are multi-factorial, it may be difficult for general physicians to diagnose dizziness. ${ }^{12}$ Therefore, the need arises for a simple scale to screen dizzy patients so as to differentially diagnose them on their first visit to the clinic itself and direct them to the appropriate mode of investigative procedures and management. ${ }^{13,14}$

There is a simple scale to screen patients and diagnose them in the first visit to the clinic and guide them to the appropriate investigative procedures and management. This scale is called Amer Dizziness Diagnostic Scale (ADDS). It helps the clinicians who are not specialized in these areas in the general clinics to evaluate and refer the patient who complains of dizziness to the appropriate specialty for further diagnosis and management. This scale is found to have a sensitivity of $96 \%$ and specificity of $96 \%$ that can adequately capture a possible diagnosis of people with vestibular disorders. ${ }^{15}$

The number of researches that study balance performance and specifically dizziness among older subjects in Saudi Arabia is limited. Thus our study is an attempt to fill the gap in the body of knowledge and in the literature about fall predictors among older population in Saudi Arabia.

\section{Patients and methods}

\section{Subjects}

Six hundred Saudi subjects including both male and females within the age group between 50 to 70 years were included in the study (Figure 1). Their demographic and baseline clinical inclusion characteristics are presented in (Table 1) (Table 2). The subjects were recruited from King Abdul Aziz University Hospital and First Clinic, Jeddah as a convenient sampling method and the study was conducted during the period of June 2014 to June 2015. The subjects who were unable to provide study information and comply with study procedures, subjects with any neuro musculoskeletal disorders, have any medical conditions which would interfere with participation in the present study, having communication difficulties, having any visual or hearing problems, and having an acute disease were excluded from the study. This study was approved by the Scientific Research Ethical Committee, Faculty of Applied Sciences, King Abdulaziz University. Informed consent was obtained from all participants.

\section{Measurements}

All participants were asked to complete answering the questionnaire that cover data related to participants previous medical history; previous fall incidence and activity level. Following which all the participants were asked to perform the required activities which are the part of Berg Balance Scale. And then each one of them were asked to answer certain questions related to the ADDS. To understand the activity level of the subjects it was used International Physical Activity Questionnaire (IPAQ) for the subjects.

\section{Berg balance scale}

Used as a test for detecting the risk of fall and composed of 14 tasks. Each item was scored from 0-4, zero means inability to do the task and four means ability to finish the task without assistance. It takes 10-20 minutes for administration. The maximal global score of the scale was 56 points. If the patient gain score ranged from 0 to 20 this means there is an impairment of balance and having a high risk of fall, whereas, if patient gain score ranged from 21 to 40 this means acceptable balance performance, and if patient gain scores ranged from 41 to 56 this means good balance performance. ${ }^{16-18}$

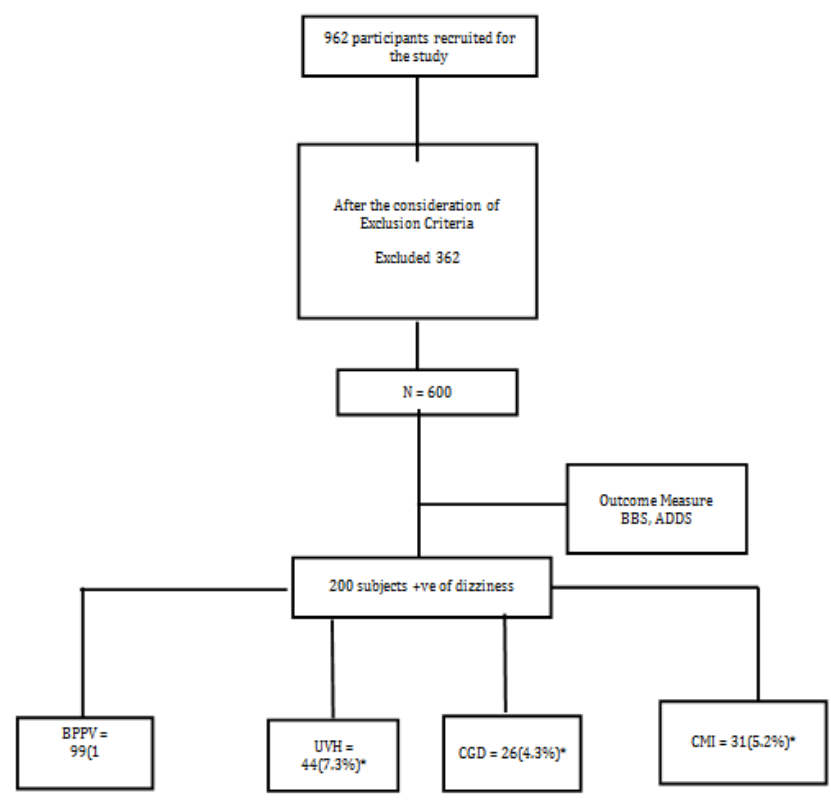

Figure I Flow chart representing the study design.

BBS, Berg Balance Scale;ADDS, Amer Dizziness Diagnostic Scale; BPPV, Benign Paroxysmal Positional Vertigo; UVH, Unilateral Vestibular Hypo-Function; CGD, Cervicogenic Dizziness; CMI, Central Mediated Impairment

*\% calculated based on total number of subjects screened

Table I Demographic characteristics of all participants $(\mathrm{N}=600)$

\begin{tabular}{|c|c|c|}
\hline & Valid & Numbers and percentage \\
\hline \multirow[t]{2}{*}{ Sex } & Male & $380(63.3 \%)$ \\
\hline & Female & $220(36.7 \%)$ \\
\hline \multirow[t]{2}{*}{ Fall History } & No & $335(55.8 \%)$ \\
\hline & Yes & $265(44.2 \%)$ \\
\hline \multirow[t]{2}{*}{ Medications } & No Medications & $285(47.5 \%)$ \\
\hline & $\geq 3$ Medications & $315(52.5 \%)$ \\
\hline \multirow[t]{2}{*}{ Activity Level } & Active & $265(44.2 \%)$ \\
\hline & Sedentary & $335(55.8 \%)$ \\
\hline \multirow[t]{2}{*}{ Ambulatory Status } & Normal & $295(49.2 \%)$ \\
\hline & With ambulatory Aids & $305(50.8 \%)$ \\
\hline
\end{tabular}

Table 2 Value of Age, Height, Weight and Berg Balance Score of all participants $(\mathrm{N}=600)$

\begin{tabular}{llllll}
\hline & No. & Minimum & Maximum & Mean & SD \\
\hline Age (Years) & 600 & 55 & 68 & 60.64 & 2.52 \\
Height (cms) & 600 & 154 & 171 & 162.4 & 8.34 \\
Weight (Kgs) & 600 & 58 & 94 & 74.38 & 13.92 \\
$\begin{array}{l}\text { Berg Balance } \\
\text { Score }\end{array}$ & 600 & 31 & 57 & 44.76 & 7.41 \\
\hline
\end{tabular}




\section{Amer dizziness diagnostic scale (ADDS)}

It consists of 17 questions arranged into five categories. The first category is general information about the patient's gender, age, history of hypertension, diabetes mellitus, balance problems; the experience of partial hearing loss associated with dizziness and the experience of blurred vision or double vision that causes vomiting. The second category is specific to Unilateral Vestibular Hypo-function (UVH), and it includes the diagnosis with a viral or bacterial infection in the last two weeks, the experience of blurred vision with or without vomiting and whether they drift to one side while walking. The third category has a critical value for Benign Paroxysmal Positional Vertigo (BPPV), and this includes the questions about the experience of dizziness while moving the head and with different body movements. Fourth category includes history of any neurological dysfunction and fifth on the time duration of dizziness (Figure 2). ${ }^{15}$ Since the ADDS was an assessment form for the differential diagnosis and for the diagnosis of dizziness which usually filled by the clinician/ Physical Therapist, due to which it was not necessary to have an Arabic version of the questionnaire.

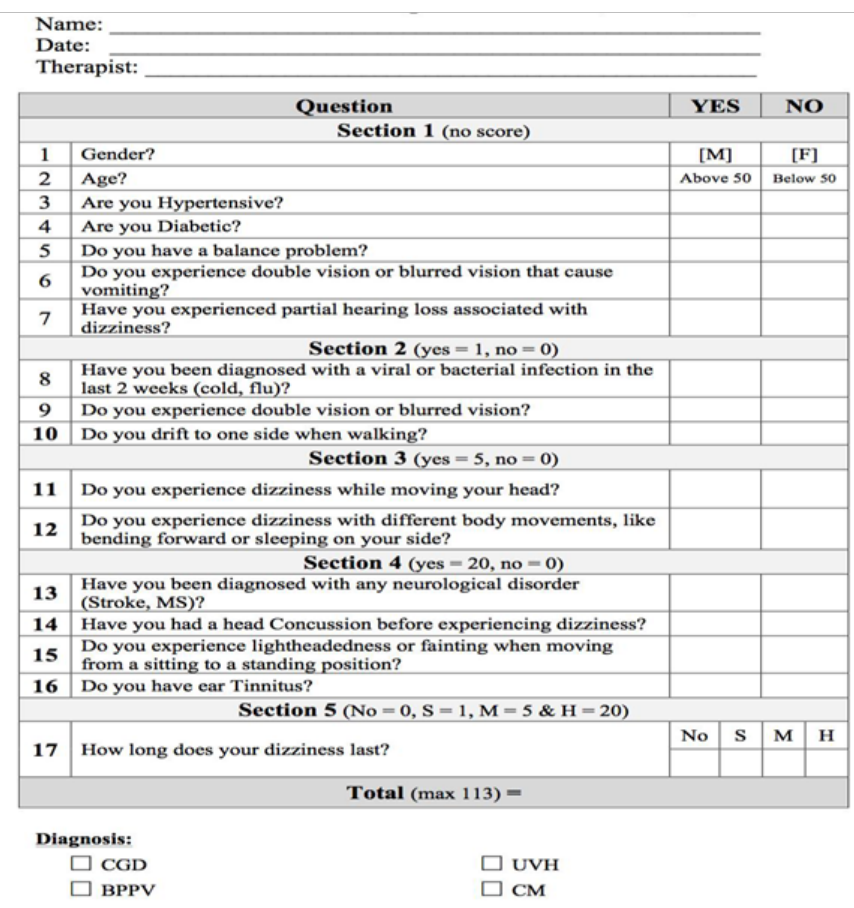

Figure 2 AMER Dizziness Diagnostic Scale (ADDS).

Even though, it was mentioned in the initial proposal to use posturography as one of the outcome tool, as we developed the ADDS which was showing a par equal results of all the objective measures used in the diagnosis of falls and dizziness, the researchers have decided to withdraw this outcome tool and continue to use the ADDS as one of the evaluative tool in the study. In the same way, the Timed Up and Go Test (TUG) also was proposed to use in the study has been withdrawn because the Berg Balance Scale was a better tool to be used for the diagnosis of fall deficits rather than TUG as this is more dependent on time rather than of functional limitations. Apart from that, BBS checks both static and dynamic balance and having more functional sub components in the evaluation form. The Video Nystagmography (VNG) was used in the previous study conducted by the same authors and found that, ADDS gives a similar findings which could be evaluated by the VNG. ${ }^{15}$

\section{Data analysis}

The collected data were analyzed using the statistical package SPSS for Windows version 19.0 (SPSS, Inc., Chicago, IL).a Frequencies and relative frequencies were computed for gender, and means and standard deviations (SD) for the continuous variables age, BBS and ADDS. Spearman's correlation and regression analysis was performed to examine the relationship among BBS and ADDS. The level of statistical significance was set at $\mathrm{p}<0.05$.

\section{Results}

The demographic inclusion characteristics of study group are presented in (Table 1) (Table 2). The majority (about 64\%) of the participants were showing a low record $(<45)$ in score in Berg Balance Scale Score, which indicates poor balance performance. Regarding the relation between the age and the Berg Balance Score, there was strong negative correlation between them $(r=-0.88, p<0.001)$ (Table $2 \&$ Figure 3 ). The number of medication intake had strong negative correlation with the Berg Balance Score $(r=-0.85, p<0.001)$. Regarding, the subjects activity level (sedentary vs. active), there was a strong direct correlation between the activity level and the Berg Balance Score $(r=0.76, p<0.001)$. Moreover, there was a strong direct correlation between the subject's ambulatory status and the Berg Balance Scale Score $(r=0.78, \mathrm{p}<0.001)$ as well.

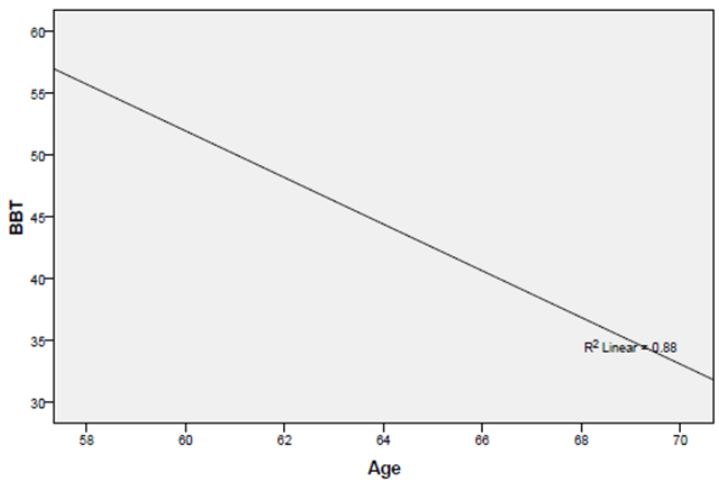

Figure 3 Linear regression between age and fall risk.

Results of the present study showed that, the ADDS revealed only 200 elderly subjects had dizziness out of the 600 elderly subjects participated in this study and the individual number of each type of dizziness are: Benign Paroxysmal Positional vertigo (BPPV) 99 [16.5\%], Unilateral Vestibular Hypo-function (UVH) 44 [7.3\%], Cervicogenic Dizziness (CGD) 26 [4.3\%] and Central Mediated Problem (CM) 31 [5.2\%] (Table 3). In addition, the results approved that the ADDS strongly correlated with the "true-positive" and "truenegative" results $(r=0.95, \mathrm{p}<0.05)$. A stepwise linear regression was conducted and the results indicated that ADDS was a significant predictor of "true-positive" and "true-negative" results (R2=0.90, p< $0.05)$.

Table 3 Number and percentage of subjects with dizziness according to ADDS $(\mathrm{N}=200)$

\begin{tabular}{ll} 
Type of dizziness & Number and percentage \\
\hline Benign Paroxysmal Positional vertigo (BPPV) & $99(16.5 \%)$ \\
Unilateral Vestibular Hypo-function (UVH) & $44(7.3 \%)$ \\
Cervico-genic Dizziness (CGD) & $26(4.3 \%)$ \\
Central Mediated Problem (CM) & $31(5.2 \%)$
\end{tabular}




\section{Discussion}

Fall is a common problem for older subjects all over the world and particularly in Saudi Arabia. ${ }^{19}$ We have conducted this study to predict the risk of fall among elderly Saudi subjects because the complications of fall are very serious especially on the elderly population. The important findings in this study shows that, there was a strong direct relationship between age and the risk of fall (87\%), i.e. the risk of fall increased with increasing of age. There are many previous studies to confirm our results. ${ }^{20-22}$ Hausdorff studied the dynamics of gait and its relation with age and concluded that, aging adversely affects the dynamic gait and induce the fall among elderly population..$^{20}$ Similarly, Al-senany studied the risk of fall among the hospitalized elderly subjects and found a high risk of fall among the majority $(62.5 \%)$ of elderly hospitalized subjects. ${ }^{21}$ Studenski and Wolter stated in their review study that, about $33 \%$ of elderly subjects above 65 years old experienced a risk of fall every year. ${ }^{22}$ In addition, Rubenstein and Josephson proved that there is a steady increase in the incidence of falls among the age group of 40-80 years old population. ${ }^{23}$

Concerning the relationship between balance with activity level and number of medications intake for associated disorders, our results proved that there was a strong direct relationship between the activity level and balance. It also shows a strong negative relationship between the number of medications intake and balance. It is well known that the majority of the elderly Saudi subjects have a sedentary life style and do little physical activities and practice low daily exercise, which leads to poor general health and increases the risk of fall. ${ }^{19}$ In addition, increasing the number of medication intake may lead to drug-drug interaction and may increase the risk of falls ${ }^{20}$ and some drugs may increase the risk of falls as a side effect of the medication. ${ }^{21,24}$

Since dizziness is highly subjective ${ }^{22}$ the components of the ADDS basically focus on subjective questions with which ultimately we can do a diagnosis, and ADDS is a clinical tool that classifies the reasons for dizziness as well. By using the ADDS, which consists of clear and simple questions, easy to understand, directed towards expected diagnosis, comprehensive questions, appropriate language and length for each questions and no bias in responses in either direction as it approved by experts who examined the scale. To our knowledge, this is the first study to develop a comprehensive scale to differentially diagnose patients complaining of dizziness, which is updated and extended to take into account of key developments in research and practice in dizziness as a first-line evaluation tool. However, a preliminary study to find out the effectiveness of ADDS in par with the VNG has been conducted earlier by the same authors. ${ }^{15}$

With the findings of the current study and the previous study, it could be recommended to consider the ADDS as a reliable reference guide for the healthcare workers, that promotes best practice care for the differential diagnosis, evaluation and treatment of dizziness. ${ }^{15}$ The most important part in the correct diagnosis of a patient with dizziness is obtaining an accurate history. However, in the absence of a valid diagnostic scale for dizziness, the clinician is put in a difficult situation where they may not be able to differentially diagnose the patients with dizziness. The results of this study indicated that the ADDS can be used in differential diagnosis of vestibular disorders. Therefore, the ADDS provides clinicians with a reliable and valid measuring tool to help in clinical decision-making process, so that even those who are not specialized in these areas can effectively screen patients who complain of dizziness and can refer them to the appropriate specialty for further diagnosis and management.

According to Jae SY et al, the preventive measures to be taken care of with utmost importance, in case if anyone detected to have a risk of fall are; awareness about the factors that may contribute for the risk of falls such as poly pharmacy, lack of care givers support and architectural barriers..$^{25}$ The future researches must be focused on the following areas like; fear of falling and the related risk factors among Saudi population, falls risk screening tool for hospital emergency departments in Saudi Arabia and factors affecting the quality of life among elderly population in Saudi Arabia.

Even though, in the initial proposal it was mentioned that, all the subjects will be assessed with the objective outcome measures, we could not conduct it on all the subjects. However, initially we have conducted it for about 48 subjects but in further we could not utilize the same for the remaining subjects mainly due to two of the following reasons

The equipment was commonly shared by many departments like; Physical Therapy, Neurology, Otolaryngolgy, and Speech \& hearing and the time allotted for each department is less. So we were not able to assess the whole number of subjects recruited for the study with this equipment. As the equipment gave some trouble in between and the $\mathrm{AMC}$ of the equipment was not renewed, we could not use the equipment for all the subjects. Thus we used the alternative outcome measure ADDS for all our subjects as this has been already mentioned in the previous reports and it's been proven its psychometric properties equivalent to the objective measures mentioned earlier. Moreover, the principal author is an expert in these area of specialty and use of ADDS.

While testing the sensitivity and specificity of any tool in statistics, it is mandatory to identify the true positive and true negative values. Here the true positive test result is one that detects the condition when the condition is present and the true negative test result is one that does not detect the condition when the condition is absent. In the current study, while testing the sensitivity and specificity of ADDS, some of the components in ADDS were related to positive and negative values and it showed exactly the same results.

One of the limitations of the study was; we were not able to use the objective outcome tools motioned in the initial proposal, however an alternative tool was used for the same instead.

The authors have conducted seminars and public awareness programs for the general public at various places of Jeddah to propagate the awareness about the risk of falls among the older population and about the preventive measures to be taken care of and thereby improve the quality of life among the older subjects in KSA.

\section{Conclusion}

The present study states that $1 / 3$ rd of the older population in Saudi Arabia are in a risk of fall which is similar to the global data ${ }^{22}$ on fall among older population. So, it is the responsibility of the authorities of health care providers and the society to address the strategies to prevent falls. In the current scenario this data is utmost important for developing strategies and providing recommendations to the stake holders regarding its importance and developing policies for fall prevention in the elderly population in Saudi Arabia. 


\section{Acknowledgements}

This project was funded by King Abdulaziz City for Science and Technology, under grant no. (AT-34-328). The researchers therefore, express their gratitude and appreciation to King Abdulaziz City for Science and Technology for providing the research grant.

\section{Conflict of interest}

Author states that there was no conflict of interest.

\section{References}

1. World Health Organisation. US National Institute of Aging. Global Health and Ageing; World Health Organisation, Geneva, Switzerland; 2011.

2. http://www.ons.gov.uk/ons/dcp171776_258607.pdf

3. http://www.who.int/healthy_settings/types/agein g/en/index.html

4. www.earthtrends.wri.org

5. Alsenany S. An exploration of the attitudes, knowledge, willingness and future intentions to work with older people among Saudi nursing students in baccalaureate nursing schools in Saudi Arabia. PhD. Thesis, University of Sheffield, United Kingdom; 2009.

6. Schöne D, Freiberger E, Sieber CC. Influence of skeletal muscles on the risk of falling in old age. Internist (Berl). 2017;58(4):359-370.

7. Donald I, Bulpitt C. The prognosis of falls in elderly people living at home. Age Ageing. 1999;28(2):121-125.

8. Wayne D, Bapp S, Grad D, et al. Temple. A Clinical Test of Stepping and Change of Direction to Identify Multiple Falling Older Adults. Arch Phys Med Rehabil. 2002;83:22-28.

9. Caslin DL, Jacobson GP. Current role of the videonystagmography examination in the context of the multidimensional balance function test battery. Semin Hear. 2009;30(4):242-252.

10. Leigh J, Zee D. The neurophysiology of eye movements.In: FA Davis, editor. Philadelphia, USA; 1983.

11. Koenig T, Duncker D, Hohmann S, et al. Clinical evaluation and risk stratification in patients with syncope. Herz. 2014;39(4):429-436.

12. Stone J, Carson A, Sharpe M. Functional symptoms and signs in Neurology: assessment and diagnosis. J Neurol Neurosurg Psychiatry. 2005;76(1):i2-i129.
13. Guerraz M1, Yardley L, Bertholon P, et al. Visual Vertigo: symptom assessment, spatial orientation and postural control. Brain. 2001;124(8):1646-1656.

14. Zainun Z, Zakaria MN, Sidek D, et al. Development of Malay Version of Vertigo Symptom Scale (MVVSS) for Clinical Use. Malays J Med and Health Sci. 2010;6(2):31-39.

15. Al Saif A, Alsenany S. Sensitivity and specificity of the Amer dizziness diagnostic scale (ADDS) for patients with vestibular disorders. $J$ Phys Ther Sci. 2015;27(1):91-96.

16. Berg KO, Wood DSL, Williams JI, et al. Measuring balance in the elderly: validation of an instrument. Can J Public Health. 1992;83(2):S7-11.

17. Berg KO, Maki BE, Williams JI, et al. A comparison of clinically and laboratory measures of postural balance in an elderly population. Arch Phys Med Rehabil. 1992;73(11):1073-1083.

18. Cimbiz A, Cakir O. Evaluation of balance and physical fitness in diabetic neuropathy patients. J Diabetes Complications. 2005;19(3):160-164.

19. Amer AlS, Ehab W, Samira A. The Prediction of Falls Among older people in Saudi Arabia. J Am Sci. 2012;8(6):692-700.

20. Hausdorff JM. Gait dynamics, fractals and falls: Finding meaning in the stride-to-stride fluctuations of human walking. Human Movement Science. 2007;26(4):555-589.

21. Alsenany S. Prevention of patient falls in a university hospital. Generations Review. 2010;20:32-45.

22. Studenski S, Wolter L. Instability and falls. In: Duthie EH, Katz PR, editors. Practice of geriatrics, 3rd ed. Saunders, Philadelphia, USA; 1998. p. 199-206.

23. Rubenstein L, Josephson K. The epidemiology of falls and syncope. Clinics in Geriatric Medicine. 2002;18(2):141-158.

24. Berdot S, Bertrand M, Dartigues J, et al. Inappropriate medication use and risk of falls - A prospective study in a large community-dwelling elderly cohort. BMC Geriatrics. 2009;9:30-42.

25. Jae SY, Chul GK, Jong EY, et al. Factors influencing falls in elderly individuals in urban and rural areas. Ageing Clin Exp Res. 2015;28(4):687-697. 